\title{
Perspectivas de la telemedicina como una alternativa para la atención en salud en Honduras
}

\author{
Reyna M. Durón ${ }^{1 *}$, Néstor Salavarría**, Heike Hesse*, Andrea Summer***, \\ Kenton Holden*** \\ *UNITEC, Tegucigalpa, Honduras. \\ **Clinica El Buen Pastor, Olancho, Honduras \\ ***Medical University of South Carolina, Charleston, USA
}

(Enviado: Noviembre, 2015. Aceptado para publicación: Marzo, 2016)

\begin{abstract}
Resumen:
La telemedicina ha demostrado tener numerosas ventajas en la atención en salud en el mundo industrializado por su aplicación, tanto para propósitos clínicos como educativos. Existe un interés creciente en la misma por dos razones: potencial de mejorar la atención en salud y reducción de costos de dicha atención. Se ha publicado varios estudios piloto sobre las posibilidades para hacer telemedicina en Centroamérica y Latinoamérica, pero el uso de la misma es escaso. Una de las aplicaciones más utilizadas ha sido la telerradiología. Las primeras experiencias con esta metodología de atención en salud en Honduras, son prometedoras y han mostrado la viabilidad de establecer redes de telemedicina que conecten al personal de salud de zonas rurales y urbanas. La integración de la tecnología de información y comunicación a los servicios de salud podría reducir costos y dar a la población mayor acceso a atención médica especializada y subespecializada. La telemedicina es un recurso innovador y eficiente en el aspecto educativo del personal de salud mediante teleconferencias virtuales o teleconsulta subespecializada con expertos en otros países.
\end{abstract}

Palabras Claves: Telemedicina; Telesalud; Consulta Remota

\begin{abstract}
:
Telemedicine has already proven to have multiple advantages for delivering timely up-to-date health care in industrialized countries by its educational as well as clinical purposes. There is a growing interest in Telemedicine apparently related to two reasons: a potential to improve quality of health care and a reduction in its costs. There have been several pilot studies on the feasibility of telemedicine in Central America and Latin America, but the use of this technology is uncommon. One of the most used applications used is teleradiology. The first experiences that we have had in Honduras are promising and have demonstrated the viability of establishing networks of telemedicine that connect health personnel from urban and rural areas. The integration of timely information and with this new health care technology could significantly reduce costs as well as increase access to specialty and subspecialty care to a larger population. Telemedicine is an innovative and efficient tool in the education of health personnel through virtual teleconferences or subspecialty teleconsultation with experts at home or abroad.
\end{abstract}

Keywords: Telemedicine; Telehealth; Remote Consultation

\section{Introducción}

\subsection{La telemedicina en el mundo}

La telemedicina ha demostrado tener numerosas aplicaciones en el mundo industrializado, tanto para propósitos clínicos como educativos (Gonzalez-Espada, 2009;

\footnotetext{
${ }^{1}$ Autor para correspondencia. Email: reynamduron@unitec.edu
} 
Franzini, 2011; Montero, 2011; Duran, 2014). El uso de la telemedicina en países en desarrollo ha mostrado potencial utilidad, pero las aplicaciones clínicas se han enfocado primordialmente en la atención para alivio de desastres (Ayyagari et al., 2003; Wooton, 2008). Recientemente en Estados Unidos se ha implementado programas para atención primaria, usando la estructura de telemedicina para atender individuos en áreas rurales y en zonas de difícil acceso.

Muchos de estos programas han mostrado tener un costo neutral o mínimo, además de índices de satisfacción favorables para el paciente y el proveedor (Crow et al., 2009; Franzini, 2011). En países subdesarrollados se ha reportado el reto de la mala conectividad del internet, la escasa banda ancha y la estabilidad de la energía eléctrica, pero el potencial de fortalecer el cuidado primario y especializado en áreas remotas a través de la telemedicina, parece vasto.

Con los avances de la tecnología, las capacidades de la telemedicina de expandirse en países en desarrollo es una meta realística. Además de conectar proveedores de salud a la distancia con especialistas, se establecen nuevas redes internas y externas con un incremento en las comunicaciones entre instituciones locales e internacionales que ofrecen servicios regionales. Estas colaboraciones pueden ser usadas para atender en afecciones agudas y crónicas, aprendizaje a la distancia y entrenamiento, dando así estímulo a los proveedores de salud que se encuentran en áreas remotas (Vassallo et al., 2001).

Otra ventaja de mejorar las colaboraciones de telemedicina dentro de un país es la mitigación de las diferencias socioculturales, incluso aquellas durante las interacciones internacionales en inglés y de norte-sur (Geissbuhler et al., 2003). Además, en las redes internas del país se reducen las barreras del lenguaje y se asume que los colaboradores van a tener un buen entendimiento del contexto local, asegurando una integración fluida de la telemedicina en la infraestructura de salud existente.

\section{La Telemedicina en Centroamérica}

La literatura médica indizada reporta algunas experiencias iniciales exploratorias de la factibilidad de la telemedicina en Centroamérica.

En Guatemala, la organización no gubernamental TulaSalud proporcionó un celular a los voluntarios de salud en una comunidad rural indígena aislada (Alta Verapaz). Gracias a los teléfonos, los voluntarios podían realizar consultas y transmitir información clínica y epidemiológica. Se comparó a este grupo con un control de voluntarios que no habían recibido teléfonos (año 2015) y se encontró que el grupo con móviles logró una reducción significativa en la mortalidad materno-infantil entre sus pacientes (Martínez-Fernández et al., 2015).

Un estudio observacional en Nicaragua (año 2014) evaluó la implementación del método POCUS (poing of care ultrasound), consistente en el uso de ultrasonidos portátiles en 132 pacientes de una comunidad rural, asistido con entrenamiento de los médicos locales vía telecomunicación por un grupo extranjero. El efecto fue un cambio en el plan de manejo inicial que se había considerado para la mitad de los pacientes (Kolbe et al., 2014).

En Panamá una unidad militar estadounidense asistió exitosamente al gobierno en el establecimiento de un proyecto demostrativo en una reserva indígena (año 2000). El proyecto se expandió hasta sumar tres clínicas rurales con servicio de telemedicina, funcionando en la actualidad con videoconferencia bidireccional en tiempo real con la 
ayuda de videófono conectado a internet, (con conexión de 768 kilobits por segundo) a un hospital local. A pesar de no contar actualmente con apoyo extranjero, a la fecha se sigue atendiendo casos de pediatría, obstetricia, neumología, dermatología y emergencias (Vega et al., 2013).

Otro reporte de Panamá incluye la experiencia de una presentación semanal de casos dermatológicos de difícil diagnóstico entre una clínica de Panamá y otra en Madrid, España (año 2012). El análisis en un estudio quasi-experimental mostró que había buena correlación entre los resultados diagnósticos de las consultas remotas y las realizadas en persona (Rios-Yul, 2012).

Existe experiencia pública y privada con la telerradiología en Centroamérica, pero se conoce pocos datos sobre su funcionalidad y eficacia (Rodríguez et al., 1998). Otros reportes de la región latinoamericana son similares en cuanto a proyectos de estudio de factibilidad y programas piloto, pero el uso de la telemedicina aún no se ha extendido en la región.

\section{Experiencia preliminar en Honduras}

La literatura médica revela que en Honduras son experiencias mínimas con telemedicina. Un estudio en 624 pacientes hondureños con enfermedades crónicas evaluó el acceso a tecnología móvil (año 2010): El 78\% de ellos poseía un dispositivo móvil (teléfono celular), $61 \%$ tenía mensajería de voz y $58 \%$ mensajería de texto. El $80 \%$ de los pacientes apreciaría asistencia para citas, recordatorios, asesoría en salud y educación a través de los móviles (Piette et al., 2010).

Una búsqueda en google reveló un segundo proyecto que involucraba el alcance de telemedicina a la región de Intibucá en Honduras, a través de una asociación con 13 centros académicos de salud en Estados Unidos, la cual se encuentra en progreso y utiliza los servidores PACS medweb (Global Med, 2014). En nuestro conocimiento, los resultados de este programa todavía no han sido publicados.

Tampoco han sido difundidas las experiencias de algunos centros privados sobre la teleconferencia educativa y la presentación de casos a distancia con médicos de otros países. Algunos centros diagnósticos han desarrollado servicios de interpretación de neuroimagen entre ciudades. En nuestra experiencia, hemos visto el beneficio de los servicios de encefalografía con interpretación a distancia hacia ciudades o comunidades rurales en Olancho, Santa Rosa de Copán y Siguatepeque. Estas experiencias en Honduras han permitido envío de reportes de estudios encefalográficos, así como apoyo a consultas de médicos sobre casos de epilepsia vía presentación electrónica de casos mediante correo electrónico.

\section{Acceso a la atención en salud en Honduras y la justificación para desarrollar opciones con telemedicina}

Honduras está ubicada en medio de Centroamérica y tiene una población de aproximadamente 7.6 millones. Es el segundo país más pobre del itsmo con una renta per cápita en el 2010 de US \$3,770. En el 2010, $58.8 \%$ de la población vivía debajo de la línea de pobreza y $36.4 \%$, en extrema pobreza (Organization, 2012). La tasa de desempleo recientemente ha alcanzado un $30 \%$. 
Según la Organización Panamericana de la Salud (OPS), en el 2008 Honduras carecía de un acceso adecuado a los servicios de salud con una tasa de cobertura de 9 médicos por cada 10,000 habitantes (Health in the Americas: Honduras, 2013). Muchos centros de salud en Honduras son atendidos por enfermeras solamente.

Aproximadamente $50 \%$ de la población vive en áreas rurales con deficiente acceso a unidades de cuidados de salud. Los indicadores de salud en la población infantil muestran que en el 2011 la tasa de mortalidad era de 18 por cada 1,000 nacimientos vivos, y la tasa en menores de 5 años era de 24/1,000, cifras significativamente más elevadas que el promedio de la región, según los datos del Banco Mundial y de la Organización Mundial de la Salud (Honduras: Health Profile, 2012).

Aunque la mortalidad en niños se asocia a infecciones principalmente, existen otras patologías de difícil diagnóstico y de manejo subespecializado que también impactan en los indicadores de morbi-mortalidad. Las teleconsultas podrían permitir a los proveedores hondureños de salud acceder a segundas opiniones nacionales o internacionales sobre diagnóstico y manejo de pacientes con diagnósticos complejos. Las redes de telemedicina en Honduras ayudarán a facilitar el acceso a los cuidados primarios y especializados. A la fecha las pocas redes con algunos componentes de telemedicina disponibles en Honduras, han facilitado el acceso a cuidado en salud, tanto primario como especializado.

\section{Educación médica por telemedicina}

Las necesidades formativas del médico se incrementan y diversifican cada vez más. En Honduras varios centros médicos e instituciones ya han iniciado la experiencia con sistemas para videoconferencias educativas para médicos. Esta necesidad continua de actualización de conocimientos, habilidades y actitudes, va a definir los nuevos modelos educativos en formación continuada, generándonos un cambio de perspectiva y potenciando exponencialmente la formación en línea, que permite capacitación en una dirección más individual y específica, más acorde a las necesidades del profesional de hoy. Esto realza la importancia de seguir buscando mejores sistemas tecnológicos para este propósito, así como ir familiarizando al personal de salud con dichos sistemas (Segura, 2016). Esto también permitirá bases para futuros sistemas digitales de telemedicina que ayudarán a mejorar el acceso de las poblaciones de escasos recursos, tanto en áreas urbanas como rurales, a la atención médica general y la subespecialidad de pediatría.

\section{Perspectivas para Honduras}

En nuestro país es factible el establecimiento de redes de telemedicina que conecten proveedores de salud, separados uno del otro por la distancia física. Los pasos para ello incluirían: la provisión de equipo con dispositivos anexos como estetoscopios digitales, cámaras y otros; soporte técnico, asegurarse de que exista capacidad de conectividad que pueda resistir un intercambio educativo bidireccional; establecer protocolos de teleconsulta con pacientes en tiempo real y evaluar la satisfacción del servicio por parte de los proveedores de salud y sus pacientes.

Aunque la calidad de los servicios de internet en Honduras puede ser una limitante, en meses recientes ha mejorado considerablemente la atención por parte de las empresas privadas proveedoras. Otro aspecto es asegurar la inversión en computadoras con suficiente capacidad de procesamiento y conexión de datos. A la par, deberá asegurarse la asistencia técnica permanente. 


\section{Aspectos éticos a considerar}

En nuestra experiencia en Honduras, es posible e importante asegurarse de lo siguiente:

- Seguridad en la red: La red debe mantenerse segura con claves de acceso y con software de protección, incluyendo programas antivirus. Cuando esté disponible, es útil el uso de intranet para comunicación entre proveedores, evitando manejar datos de pacientes en correos electrónicos normales.

- Privacidad y confidencialidad de la información de pacientes: se debe manejar prudentemente los archivos digitales, incluso se puede utilizar la anonimización de datos en caso de transmisiones internacionales o cuando se considere pertinente.

- Consentimiento informado: es importante utilizar este instrumento y contar con la monitoría de los comités de ética de las instituciones participantes. En el caso de adolescentes se podría utilizar una hoja de asentimiento.

- Beneficios al paciente: El paciente siempre será beneficiado de las discusiones de caso. Sin embargo deberá señalarse la responsabilidad legal de las segundas opiniones. Deberá aclararse que la consulta a distancia puede tener algunas desventajas respecto a la consulta presencial. La participación en una teleconferencia de interconsulta médica internacional no significa ningún compromiso de manejo médico por parte de los consultados; el manejo del paciente deberá seguir a cargo del médico tratante que refiere el caso por escrito.

- Capacitación ética de proveedores: Todos los participantes en las teleconsultas deberán ser capacitados sobre la privacidad y confidencialidad del servicio, dejando este compromiso por escrito.

\section{Conclusión}

La telemedicina ha demostrado tener numerosas aplicaciones en el mundo, pero en Honduras la experiencia es reciente y limitada. Muchos pacientes viven dificultades de acceso geográfico a atención especializada en salud y los médicos en atención primaria viven dificultades por el difícil acceso a interconsultas con especialistas y a educación médica continua. La conexión mediante redes de telemedicina en Honduras sería una opción, tanto para educación médica como para atención directa de pacientes. Se requiere en primer lugar poner a prueba las tecnologías de información y comunicación, y protocolos de trabajo de redes a nivel interciudad e internacional para poder desarrollar la telemedicina en Honduras. En un estudio piloto, hemos encontrado la factibilidad y satisfacción de los usuarios en una red de telemedicina entre Olancho y Tegucigalpa (Honduras) y Carolina del Sur (Estados Unidos), comprobando que dicha red es posible y de gran utilidad para consultas intranacionales e internacionales (Brooks et al., 2016). Este proyecto se estará expandiendo en los futuros meses.

\section{Referencias}

Ayyagari A, et al. (2003). Use of telemedicine in evading cholera outbreak in Mahakumbh Mela, Prayag, UP, India: an encouraging experience. Telemed J E Health, 9: 89-94. 
Brooks M, et al. (2016). Feasibility of developing a pediatric telehealth network in Honduras with international consultation support. Rural and Remote Health (en revisión).

Crow SJ, et al. (2009). The cost effectiveness of cognitive behavioral therapy for bulimia nervosa delivered via telemedicine versus face-to-face. Behav Res Ther, 2009. 47(6): p. 451-3.

Duncan C, et al. (2010). Safety and effectiveness of telemedicine for neurology outpatients. Scott Med J, 55: 3-5.

Franzini L, et al. (2011). Costs and cost-effectiveness of a telemedicine intensive care unit program in 6 intensive care units in a large health care system. J Crit Care, 26(3): 329 e1-6.

Geissbuhler A, et al. (2003). Telemedicine in Western Africa: lessons learned from a pilot project in Mali, perspectives and recommendations. AMIA Annu Symp Proc, 2003: 249-253.

Global Med. USDLA Awards Submission [cited 2014 3-12-14]; Available from: http://www.globalmed.com/about-globalmed/usdla-awards-submission.php.

Gonzalez-Espada WJ, et al. (2009). Achieving success connecting academic and practicing clinicians through telemedicine. Pediatrics, 123: e476-83.

Health in the Americas: Honduras. 2013 4/8/13]; Available from: http://new.paho.org/saludenlasamericas/index.php?option=com_content\&view=ar ticle\&id=43\&Itemid=50\&lang=en.

Honduras: health profile. May 2012 4/8/13]; Available from: http://www.who.int/gho/countries/hnd.pdf.

Kolbe N, et al. (2014). Point of care ultrasound (POCUS) telemedicine project in rural Nicaragua and its impact on patient management. J Ultrasound, 18: 179-185.

Martínez-Fernández A, et al. (2015). TulaSalud: An m-health system for maternal and infant mortality reduction in Guatemala. J Telemed Telecare, 21(5): 283-291.

Monteiro A.M, et al. (2011). Telemedicine and pediatric radiology: a new environment for training, learning, and interactive discussions. Telemed J E Health, 17: 753-756.

Organization, W.H. Country Cooperation Strategy at a glance: Honduras. 2012 4/5/13]; Available from: http://www.who.int/countryfocus/cooperation_strategy/ccsbrief_ hnd_en.pdf.

Piette JD, et al. (2010). Access to mobile communication technology and willingness to participate in automated telemedicine calls among chronically ill patients in Honduras. Telemed J E Health, 16: 1030-1041.

Ríos-Yuil JM. (2012). Correlation between face-to-face assessment and telemedicine for the diagnosis of skin disease in case conferences. Actas Dermosifiliogr, 103:138143.

Rodríguez RI, et al. (1998). Teleradiology. The experience in Panamá. Rev Med Panama, 23: $10-14$.

Segura Badia H. (2006). Las nuevas tecnologías y la formación médica continuada a distancia. Educ Méd, 9: 22-30. 
Vassallo DJ, et al. (2001). An evaluation of the first year's experience with a low-cost telemedicine link in Bangladesh. J Telemed Telecare, 7: 125-138.

Vega S, et al. (2013). Testing a top-down strategy for establishing a sustainable telemedicine program in a developing country: the Arizona telemedicine programUS Army-Republic of Panama Initiative. Telemed J E Health, 19: 746-753.

Wootton R. (2008). Telemedicine support for the developing world. J Telemed Telecare, 14: $109-114$.

"LA REVISTA INNOVARE NO SE HACE RESPONSABLE EN NINGÚN CASO DE LOS CONTENIDOS, DATOS, CONCLUSIONES U OPINIONES VERTIDAS EN LOS ARTÍCULOS PUBLICADOS, SIENDO ESTA RESPONSABILIDAD EXCLUSIVA DEL (DE LOS) AUTOR (AUTORES)" 